\title{
Nursing students' clinical judgment in high-fidelity simulation based learning: A quasi-experimental study
}

\author{
Hao Bin Yuan", Beverly A. Williams², Chan Yok Man ${ }^{1}$ \\ 1. School of Health Sciences, Macao Polytechnic Institute, Macao. 2. Faculty of Nursing, University of Alberta, Edmonton, \\ Alberta, Canada.
}

Correspondence: Hao Bin Yuan. Address: Rua de Luis Gonzaga Gomes, Macao. Email: haobinfriend@163.com

Received: November 28, 2013 Accepted: January 20, $2014 \quad$ Online Published: February 18, 2014

DOI : $10.5430 /$ jnep.v4n5p7

URL: http://dx.doi.org/10.5430/jnep.v4n5p7

\begin{abstract}
Background: Improving students' decision making and judgment skills becomes an essential part of nursing education. The purpose of this Quasi-experimental study was to assess nursing students' clinical judgment in high-fidelity simulation based learning using observational measures.

Method: Based on Tanner's clinical judgment model, a single group repeated-measures design was used with a purposive sampling at one nursing school in Macao. A total of 113 baccalaureate nursing students (49 in year two, 64 in year three) completed five simulation sessions within a 36 hour period. Two tutors used the Clinical Judgment Rubric to assess students' clinical judgment at the end of each simulation session. The inter-rater reliability for the sessions ranged from 0.833 to 0.910 .

Results: The scores for clinical judgment increased from the first simulation scenario to the last scenario. Compared with third-year students, second-year students had significantly higher scores of clinical judgment in scenarios two, three, four and five. The debriefing was essential to preparing students for improving their thinking and reasoning skills. Students perceived that simulation strengthened their theoretical knowledge and helped them to notice, interpret, and respond appropriately to contingencies and emergencies. Tutors' comments indicated that second-year students valued the newly learned knowledge and used it to analyze the simulated scenarios while third-year students presented less knowledge and skill preparation.

Conclusions: High-fidelity simulation using a computer-controlled human patient simulator has potential to support the development of clinical judgment in nursing students. Tutors are challenged to enhance students' intrinsic motivation for learning, and encourage them to continue their efforts in learning. Further research is needed to explore whether senior students have better performance in clinical judgment than junior students.
\end{abstract}

\section{Key words}

Clinical judgment, Baccalaureate, Nursing students, High-fidelity simulation

\section{I ntroduction}

Clinical judgment is defined as an interpretation and reaching a conclusion about a patient's situation and the decision by the nurse to intervene. It requires that nurses consider a variety of complex factors in order to make the appropriate 
decisions which are specific to each patient situation ${ }^{[1]}$. Nurses need to utilize their professional knowledge and clinical experience in order to recognize subtle variations in clinical situations, interpret what they observe, and decide how to provide nursing care for individual patients. Clinical judgment is an essential skill in caring for patients. Improving the quality of nursing care depends on enhancing nurses' judgment and decision making skills ${ }^{[2]}$. Well-developed observational and reasoning skills are necessary to make sound and reliable clinical judgments. Therefore helping students develop decision making and judgment skills becomes an essential part of effective nursing education. When actual patient learning situations are not readily available, teachers can create simulated clinical learning situations for their students. The use of simulated situations allows students to apply theoretical knowledge to analyze clinical situations systematically, reason situations analytically and develop clinical judgment through the integration of critical thinking and clinical reasoning ${ }^{[3]}$.

As an interactive learning method, simulation involves imitation of real patient experiences that can be used for teaching and practicing personal and interpersonal abilities such as clinical judgment, communication, prioritizing and time management ${ }^{[4]}$. Human patient simulators (HPS) have been used to provide a realistic situation with physiological functions under the control of the computer software. Students can address a learning issue "as if it is", and make, detect and correct patient care errors without negative consequences. Simulation becomes the way to promote the development of clinical reasoning and judgment and facilitate deeper learning and knowledge transformation ${ }^{[5,6]}$.

Chinese education has been influenced by Chinese culture and Confucianism. The process of learning emphasizes the significance of reflective thinking which requires a spirit of inquiry and open-mindedness. Chinese students have been socialized to respect the wisdom of their teachers who provide the knowledge and guide them to make decisions. Teaching proceeds in an orderly way with specific examples to explain the concepts. Effective learning actually occurs in a complex and interactive environment, and conceptions of learning and knowing are deeply rooted in specific cultural contexts. Therefore, there is a need to reappraise traditional teaching methods. Preparation of students for a complex health care environment requires that students should learn how to analyze situations, interpret problems and make decisions for solving the problems. In this case, scenario-based and/or problem-orientated with active learning strategies, such as simulation or experience learning should be promoted to foster students' clinical judgment. Simulations on static manikins under the direction or demonstration of teachers have commonly been used. More recently, interactive high-fidelity simulation is being used to facilitate not only skill learning but also critical thinking and clinical judgment.

A systematic review on current empirical studies (between 2000 and 2011) indicated the high-fidelity simulation did enhance scores on knowledge and skill exams but provided a mixed contribution to self-confidence and competency ${ }^{[7,8]}$. Based on self-report surveys, many Chinese students perceived that simulation effectively improved their abilities in communication, team collaboration, clinical thinking, clinical judgment and problem solving ${ }^{[9,10]}$. However, clinical judgment is particularistic and dependent on the unique situation, so the validity of any general self-report measures becomes questionable. Lasater (2007) ${ }^{[11]}$ suggested that high-fidelity simulation provides a comprehensive situation for tutors to assess students' clinical judgment and determine their recognition of gaps in understanding patient situations, but there is no empirical evidence using an observational measure to support the contribution of high-fidelity simulation to students' clinical judgment.

\subsection{Purpose of the study}

The purpose of this study was to assess nursing students' clinical judgment in high-fidelity simulation based learning using observational measures. The research question was: Is there a difference in clinical judgment, as measured by the clinical judgment rubric through a series of simulation sessions?

\subsection{Conceptual framework of the study}

Based on Tanner's model, clinical judgment incorporates noticing, interpreting, responding and reflection. Noticing is interpreted as having a sufficient understanding of the situation while interpreting is making meaning of the available data 
in a specific clinical situation. Three interrelated reasoning patterns of thinking (analytic reasoning, intuition, narrative thinking) are used to interpret or make sense of a patient's situation. Analytic reasoning is a hypothetical-deductive process which is used to generate possible causes and impacts of each plausible problem. Intuition is an immediate sense of apprehension in response to some unusual situation and narrative thinking creates an inclusive background for understanding a patient's unique situation and helps the students set up priorities for clinical judgments being made. Responding involves developing an appropriate intervention for dealing with the problem and reflecting refers to a reviewing the outcomes of the action and determines whether the expected goals were achieved or not. Reflection includes both reflection-in-action and reflection-on-action ${ }^{[1]}$. Reflection-in-action is used to determine how the patient is responding to nursing interventions and involves adjusting the interventions based on the reassessment of patient's condition. Reflecting-on-action involves critical self-analysis of the identification of decision points, and acknowledgement of strength and weakness in both skill performance and reasoning patterns. In simulation, students are exposed to the simulated scenario and initially determine what is happening with the patient. When students recognize an unexpected or abnormal condition, they pursue additional information through health assessment and history inquiries. Then students develop the care plan and identify nursing interventions through group discussion. Finally students reflect on what has happened, how they responded, and what needs to be improved in the next session. The integration of feedback and reflection increase the students' abilities to synthesize knowledge from multiple sources and make sound and safe decisions ${ }^{[11]}$. The conceptual framework for the development of clinical judgment in simulation is shown in Figure 1.

\section{Method}

\subsection{Design}

A quasi experimental study was conducted with a single group repeated-measures design at one nursing school in Macao.

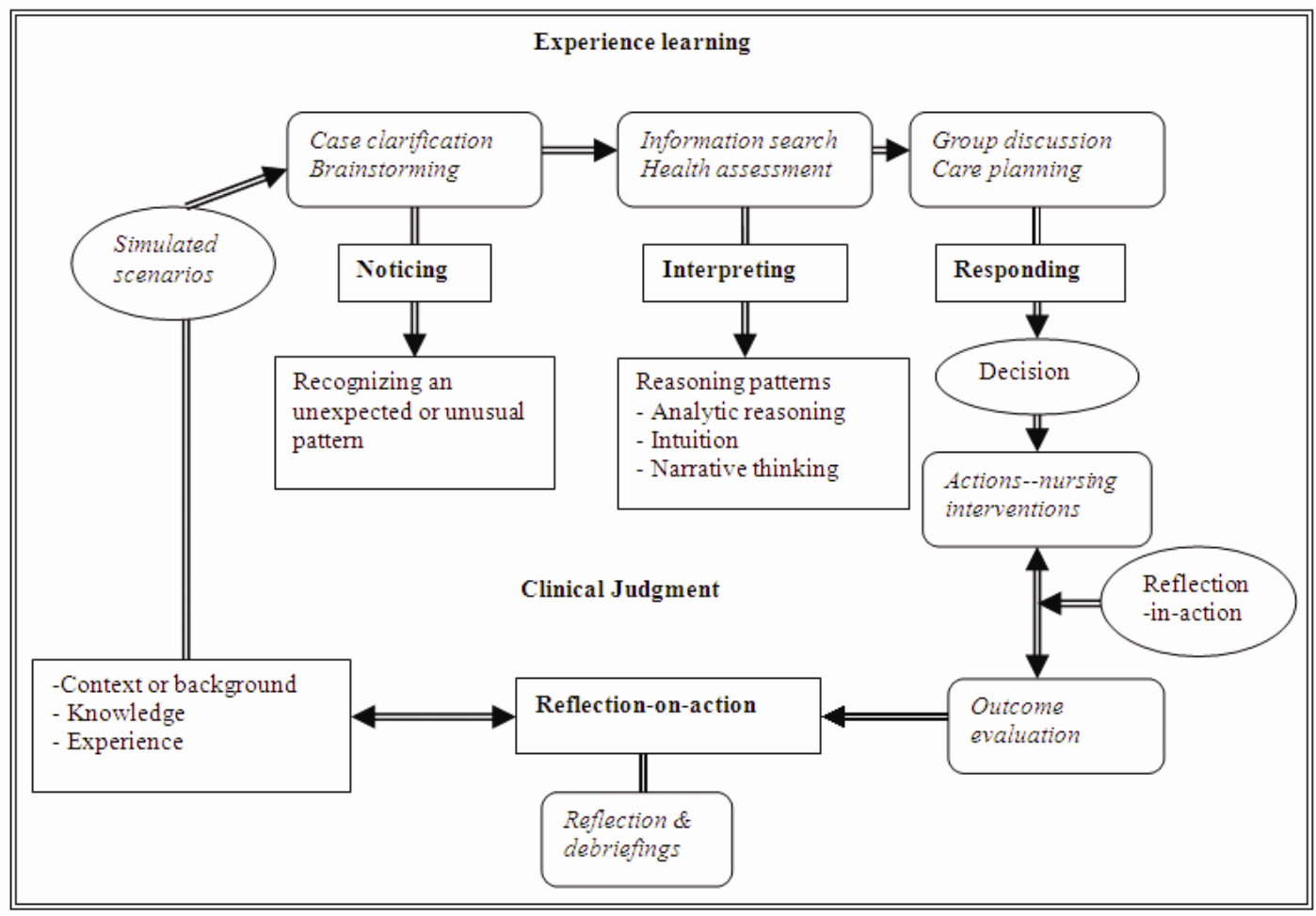

Figure 1. Conceptual framework for the development of clinical judgment in experience learning 


\subsection{Sample}

A purposive sampling was used to invite all year two and year three baccalaureate nursing students who had completed courses in fundamentals of nursing, health assessment and medical-surgical nursing to participant in this study. Informed consent was obtained. Although 120 students voluntarily enrolled in this study, 113 students (49 in year two, 64 in year three) completed all sessions. The average age was 21.51 (S.D. 1.31) years old.

\subsection{The interaction with the computer-controlled simulator}

The scenarios were designed using the high-fidelity simulator which is a computer-controlled human patient simulator (HPS, SimMan). The SimMan is a full-body manikin with a realistic upper airway, chest movement, variable cardiac and breath sounds and a palpable pulse, an ability to verbal some symptoms and respond to interventions. Five different simulated scenarios were used: Patient A a 42 year-old man with appendicitis; Patient B a 68-year old man, with chronic obstructive pulmonary disease; Patient C a 73-year-old man with gastrointestinal bleeding; Patient D a 72-year old man with myocardial infarction; Patient E a 32-year old man with critical trauma. Students were assigned to take part in five separate immersive high- fidelity simulation scenarios one after another, beginning with Patient A and ending with Patient E over 36 learning hours. Each learning experience was completed within 6 to 8 learning hours. In each year, 5 to 6 students worked as a single group and engaged in the learning activities under the guidance of two qualified tutors.

Students received an orientation to the simulation laboratory to familiarize them with the technology and the simulation format. The learning process includes case clarification, health assessment, nursing intervention and debriefing. In each session, the students directly interacted with a contextual patient scenario. Students are given a brief amount of time to review the scenarios, clarify what had happened with the patients, and discuss the approach to care. During the enactment of the scenario, the students were expected to demonstrate an emotional connection and relationship with the patient to enhance realism. They performed assessments and interventions using appropriate techniques and adhering to principles of safety. Students were concerned with the main patient concerns, including the provoking and relieving factors. They conducted a general assessment including consciousness, vital signs, skin, sensory, cardiovascular, respiratory, abdominal, musculoskeletal, and sensory assessments. They needed to be able to recognize any abnormal signs and symptoms, and provide appropriate explanations and health education for the clients. They also completed the health history inquiry including medications, allergies, previous surgery and family history. In the meantime, they have to manage any concern of the clients, such as pain, breathless, vomiting, nausea, cough, thirst and hunger. After they assessed the patients and reviewed the laboratory data, students were required to think about the priority of the problems, and implement appropriate ordered interventions including medication administration, blood transfusions, wound care, urethral catheterization, oxygen therapy, electrocardiogram exam while observing the patients' physiological responses and reassessing the patient condition. Sometimes they encountered a critical situation such as severe arrhythmia, bleeding or breathless. When they did encounter such situations, they were expected to intervene appropriately and within their scope of practice. The situation would often mean calling the physician for additional orders. If they made a wrong decision, the patient's condition became worse and in some cases resulted in death. At that time, the tutors encouraged reflection on their performance and helped them identify the possible mistakes while encouraging them to discuss how they might have modified their assessment and intervention. The simulation was repeated so that students had a chance to make a right decision. In this way, students learned more from their mistakes and realized the importance of safe practice. A debriefing session followed each scenario in the simulation laboratory. It focused on team care of the patient in terms of safe practice, priority setting, continuous assessment, communication and resource management. The debriefing questions included: (1) What were the key concepts and skills you used in this session? (2) What do you need to learn more about in order to take care of patients in similar situations? (3) What needs to be improved in the next session? 


\subsection{I nstrument}

Based on Tanner's clinical judgment model, Lasater (2007) ${ }^{[11]}$ developed a checklist of clinical judgment, the Lasater Clinical Judgement Rubric (LCJR), to guide raters in using an observational measurement. The LCJR is used to evaluate student' behaviors of clinical judgment which includes noticing ( 3 items), interpreting ( 2 items), responding (4 items) and reflecting ( 2 items) along a continuum where 1 = beginning, 2 = developing, 3 = accomplished, and or 4 = exemplary. The total score on the LCJR can range from 11 to 44 . A higher score indicates better performance in clinical judgment.

In this study, the LCJR was used by two qualified tutor raters in assessing the performance of each group. To control for rater bias, the inter-rater reliability of LCJR was established in each session. The inter-rater reliabilities of the LCJR were 0.833 (session 1, $P<.01$ ), 0.878 (session 2, $P<.01$ ), 0.839 (session $3, P<.01$ ), 0.869 (session $4, P<.01$ ) and 0.910 (session $5, P<.01$ ). In addition, each group was invited to discuss how simulation experience works on their clinical judgments for understanding students’ perceptions about the development of clinical judgment during simulation.

\subsection{Date collection and analysis}

Each group simulation was video-recorded, which provided the opportunity to use the rubric and practice evaluating students' performance and clinical judgment. Students' clinical judgment was assessed in each simulation session prior to debriefing. Upon careful review of the LCJR, raters defined the scoring categories and differences between score categories. The raters completed the scoring for each group and provided the rationale for each score assigned. Differences in interpretation were discussed and appropriate adjustments to the rubric were negotiated until similar rationale for scores given could be verbalized. The average scores of two raters were calculated. The repeated measures analysis of variance (RM-ANOVA) was used to assess differences of clinical judgment within groups as well as across time for simulation. Independent sample t-tests were carried out to compare the mean scores of the LCJR between year two and year three students.

Each group discussion took place in the simulation laboratory once students had completed all sessions. The researcher emphasized the need for confidentiality and obtained written consent from all participants. Discussions were tape-recorded and transcribed verbatim for data collection and analysis, and lasted approximately 15 minutes. The participants were encouraged to fully describe their thoughts. Credibility was ensured through consultation with participants and their revisions to their transcripts. Two researchers individually and repeatedly read each transcription line by line to become immersed in the content, and then the data were summarized based on the emerging meanings. The two researchers compared their results. The differences were discussed until consensus was achieved. Finally, all transcriptions were reviewed again to ensure that all data had been included and analyzed.

\subsection{Ethical considerations}

This study was discussed by the workgroup for Academic Affaires and approved by the Board of Management of the institute in terms of research process, ethical consideration and human rights protection. All participants were informed with a complete explanation about the nature of the study and all signed the consent inform. Participation was voluntary, and students had the freedom to withdraw from the study at any time. Confidentiality and autonomy were assured throughout the study. Participants were informed that only aggregate data would be reported.

\section{Results}

Chinese students' clinical judgment increased from the first session to the last session which suggests that the five simulation experiences were helpful in developing clinical judgment in helping students to notice, interpret, respond and reflect (see Table 1). It was also interesting to find that second-year students consistently achieved higher scores in all elements of clinical judgment than did third-year students (see Table 2). 
Table 1. Changes in clinical judgment through simulation sessions $(\mathrm{N}=113)$

\begin{tabular}{|c|c|c|c|c|c|c|}
\hline \multirow{2}{*}{ Clinical Judgment } & \multicolumn{5}{|l|}{ Mean (S.D.) } & \multirow{2}{*}{$\begin{array}{l}\text { RM-ANOVA } \\
\text { Greenhouse-Geisser Value }\end{array}$} \\
\hline & Session 1 & Session 2 & Session 3 & Session 4 & Session 5 & \\
\hline Noticing & $5.48(1.42)$ & $5.58(1.06)$ & $6.23(1.21)$ & $6.88(1.47)$ & $7.36(1.29)$ & $\begin{array}{l}F=50.753 \\
P=0.000\end{array}$ \\
\hline Interpreting & $3.73(0.86)$ & $3.76(0.95)$ & $4.39(0.84)$ & $4.71(0.93)$ & $5.09(0.84)$ & $\begin{array}{l}F=60.649 \\
P=0.000\end{array}$ \\
\hline Responding & $6.31(1.36)$ & $6.37(1.51)$ & $7.15(1.41)$ & $8.13(1.61)$ & 8.69 (1.93) & $\begin{array}{l}F=71.761 \\
P=0.000\end{array}$ \\
\hline Reflecting & $3.78(0.96)$ & $3.35(0.93)$ & $3.97(1.03)$ & $4.14(1.11)$ & $4.75(1.15)$ & $\begin{array}{l}F=41.406 \\
P=0.000\end{array}$ \\
\hline Overall score & $19.30(4.13)$ & 19.06 (3.87) & 21.74 (3.98) & $23.87(4.68)$ & $25.89(4.72)$ & $\begin{array}{l}F=71.693 \\
P=0.000\end{array}$ \\
\hline
\end{tabular}

Table 2. Comparison of the scores of clinical judgment between year two and year three students

\begin{tabular}{|c|c|c|c|c|c|}
\hline \multirow{2}{*}{ Clinical Judgment } & \multicolumn{5}{|l|}{ Mean (S.D.) } \\
\hline & Session 1 & Session 2 & Session 3 & Session 4 & Session 5 \\
\hline \multicolumn{6}{|l|}{ Noticing } \\
\hline Year 2 & $5.27(1.06)$ & $5.84(0.66)$ & $6.83(1.27)$ & $8.00(0.92)$ & $8.09(1.16)$ \\
\hline Year 3 & $5.65(1.63)$ & $5.38(1.26)$ & $5.78(0.94)$ & $6.02(1.21)$ & $6.81(1.09)$ \\
\hline \multirow{2}{*}{ Independent samples $t$-test } & $t=-1.509$ & $t=2.333$ & $t=5.073$ & $t=9.487$ & $t=6.002$ \\
\hline & $P=.134$ & $P=.013$ & $P=.000$ & $P=.000$ & $P=.000$ \\
\hline \multicolumn{6}{|l|}{ Interpreting } \\
\hline Year 2 & $3.80(0.49)$ & $4.26(0.613)$ & $4.67(0.88)$ & $5.29(0.57)$ & $5.57(0.37)$ \\
\hline Year 3 & $3.69(1.07)$ & $3.38(0.99)$ & $4.18(0.75)$ & $4.27(0.93)$ & $4.72(0.91)$ \\
\hline \multirow{2}{*}{ Independent samples $t$-test } & $t=0.720$ & $t=5.718$ & $t=3.150$ & $t=7.140$ & $t=6.217$ \\
\hline & $P=.473$ & $P=.000$ & $P=.002$ & $P=.000$ & $P=.000$ \\
\hline \multicolumn{6}{|l|}{ Responding } \\
\hline Year 2 & $5.92(1.12)$ & $7.03(0.81)$ & $8.10(1.21)$ & $9.36(1.28)$ & $10.06(1.36)$ \\
\hline Year 3 & $6.60(1.46)$ & $5.87(1.71)$ & $6.42(1.08)$ & $7.19(1.14)$ & $7.20(1.14)$ \\
\hline \multirow{2}{*}{ Independent samples $t$-test } & $t=-2.806$ & $t=4.390$ & $t=7.795$ & $t=9.343$ & $t=8.500$ \\
\hline & $P=.006$ & $P=.000$ & $P=.000$ & $P=.000$ & $P=.000$ \\
\hline \multicolumn{6}{|l|}{ Reflecting } \\
\hline Year 2 & $3.89(0.68)$ & $3.91(0.68)$ & $4.72(0.82)$ & $5.04(0.82)$ & $5.71(0.85)$ \\
\hline Year 3 & $3.70(1.12)$ & $2.92(0.86)$ & $3.39(0.77)$ & $3.45(0.76)$ & $4.01(0.73)$ \\
\hline \multirow{2}{*}{ Independent samples $t$-test } & $t=1.129$ & $t=6.797$ & $t=8.895$ & $t=10.515$ & $t=11.465$ \\
\hline & $P=.262$ & $P=.000$ & $P=.000$ & $P=.000$ & $P=.000$ \\
\hline \multicolumn{6}{|l|}{ Overall score } \\
\hline Year 2 & $18.87(2.78)$ & $21.03(2.21)$ & $24.33(3.76)$ & $27.68(3.19)$ & $29.44(3.22)$ \\
\hline Year 3 & $19.63(4.91)$ & $17.55(4.18)$ & 19.77(2.87) & $20.95(3.33)$ & $23.16(3.78)$ \\
\hline \multirow{2}{*}{ Independent samples $t$-test } & $t=-1.046$ & $t=5.288$ & $t=7.057$ & $t=10.849$ & $t=9.285$ \\
\hline & $P=.298$ & $P=.000$ & $P=.000$ & $P=.000$ & $P=.000$ \\
\hline
\end{tabular}

Additionally, the data from 21 group discussions indicated that students perceived that simulation enabled them to strengthen their theoretical knowledge allowing them to understand what was happening with the patient in order to make an appropriate judgment. They explained:

'Simulation mimicked what I have been learning.' 
'I applied the previous knowledge to analyze the data... it helped me to understand the patient's needs....recognize some abnormal signs and symptoms.'

'Practicing the realistic scenarios helped us to interpret the patient's concerns...and make sense of what we learned...'

'...we pull our knowledge and skills together for analyzing clinical problems while making a appropriate judgment...'

Students considered that simulation helped them to notice, interpret, and respond appropriately to contingency and emergency situations. They said:

'The realistic scenarios guided us to search for advanced information, and explain why the patients have those kinds of symptoms and signs...'

'...it helped us to improve our thinking and reasoning skills: for example, we explained the patients' condition promptly and provided appropriate patient education for the patient while assessing the patient.'

Furthermore, simulation helped them to define the patient's needs and problems, and make a decision to engage in appropriate interventions. For example:

'...the patient coughs frequently and complains of severe wound pain while we are doing wound dressing for the patient after the operation. We protect the wound with sterile gauze first ... as well as check the vital signs and the repertory sound, then we discuss the potentials of the patient's condition and made a decision...'

'...patient presents coughing and gasping... we immediately check the breath sounds....the wheeze in both sides of the lung... the patient was treated by anti-asthmatic drugs following a medical order, then we check the oxygen saturation again ....and decide whether to give the oxygen to the patient.'

The debriefing was essential to preparing students for improving their thinking and reasoning skills. They said:

‘...debriefing helped us realize what went well ... what didn’t... everyone was supportive .... I definitely learned from the feedback...'

'...debriefing let us to reflect on our performance....and developed our thinking and reasoning skills when we gave the comments to others'

'...Peers' feedback gave me some new perspectives...it is very helpful for increasing my reasoning skills.'

\section{Discussion}

The quantitative and qualitative findings suggested that simulation using HPS helped Chinese students to transform classroom knowledge and skills for use in clinical situations and facilitated the development of students' clinical reasoning and thinking skills and judgments. The scenarios were designed to create various kinds of complex and ambiguous problems that required students to make accurate clinical judgments. Students engaged in narrative reasoning. They assessed the patient, recognized significant aspects of an undefined clinical situation, and used narrative thinking to interpret the meaning of data, used analytic thinking processes to generate alternatives, weigh alternatives against the evidence rationally, finally they had rationale for judgments and chose the most appropriate response to the circumstances. This recognition process enhanced their clinical reasoning. Narrative reasoning helped students direct their attention to the patients' physical issues as well as understand patients' emotional needs and possible resources to assist then in coping with the situation ${ }^{[12]}$. The interaction with realistic scenarios enabled students to "read" the patient's responses to the nursing intervention and adjust the interventions based on the data from reassessment. Clinical judgment was increased by assessment of the relevant data, meticulous analysis of the detailed data, and a logical interpretation and reasoning. 
Students utilized specific knowledge to solve problems, accumulating new knowledge through their simulation experience. They consistently identified the knowledge they used to assess the situation, and recognized how they used the knowledge in interpreting data. When students did not identify the tacit knowledge or applied it incorrectly, tutors guided them in realizing their mistakes and correcting them. Simulation promoted the development of a deeper understanding related to the patient issues ${ }^{[4]}$. The debriefing questions were designed to help students assess their own knowledge and develop reflective practices. Debriefing facilitated reflective thinking both individually and collectively among peers with the intent of transforming the simulation experience into learning. The debriefing focused on clarifying the students' misunderstandings and facilitating in-depth understanding of theoretical knowledge used in scenarios. Students were guided to realize their mistakes and notice the relevant factors and lack of clinical knowledge related to a particular situation. The recognition of reasoning patterns helped students analyze why they made premature conclusions without sufficient data or what they needed to learn more about. Reflection-on-action showed that what students gain from their experience contributes to the development of relevant knowledge and clinical skills. Reflection on practice is critical for the development of clinical knowledge and improvement in clinical reasoning. Debriefing can facilitate the application of clinical judgment through reflective learning ${ }^{[1,6]}$.

The findings of this study are supported by previous studies. Rhodes and Curran (2005) ${ }^{[13]}$ suggested that human patient simulators can help students enhance knowledge, facilitate skill acquisition, decrease anxiety, and promote clinical judgment in a safe environment. Active participation in a variety of clinical scenarios is an enjoyable way to strengthen the student's ability to make appropriate clinical decisions. Simulation experiences can be beneficial in enhancing student confidence regarding patient care decisions, and progressing to the advanced stage of practice. Nielsen et al. (2007) ${ }^{[14]}$ used the clinical judgment mode to guide students' reflection and found that a structured guided reflection can potentially enhance thinking and move students toward increased nursing competence as well as facilitate progression in the development of student thinking and clinical judgment. The combination of experiential learning and guided reflective practices provided a learning environment for the development of clinical judgment ${ }^{[1]}$. Kienle and Kiene (2011) ${ }^{[3]}$ concluded that the knowledge required in clinical judgment grows with experience, and aided in instantaneous recognition of repeat clinical situations.

Clinical judgment originates from the nurse's theoretical knowledge, practical experience and ethical perspectives. Clinical judgment is influenced substantially by individual background and pre-existing knowledge and experience ${ }^{[1]}$. The interesting finding in this study was that sophomores had better clinical judgment than third-year students. It may be caused by the different levels of engagement. Tutors' comments showed that second-year students valued the newly learned knowledge and used it to analyze the simulated scenarios. They engaged in active learning and deep discussions. So they had greater achievement in clinical reasoning and judgment. However, third-year students did not seem to have a deep understanding of some of the knowledge that they learned in their previous two years, and did not do a full knowledge review before the simulated learning experience. Nursing assessment was not comprehensive. The interpretations to abnormal signs and symptoms were ambiguous. Sometime third year students could not recognize severe arrhythmias. Therefore, although their clinical judgment did increase with the simulation sessions, there was less improvement when compared with the sophomores. It suggests that tutors should consider how to promote students' intrinsic motivation for learning, develop their potential and encourage them to keep their efforts in learning as they progress through the nursing education program.

\section{Limitations of this study}

The generalization of the findings to all Chinese nursing students was limited because of small sample size from one school of nursing and one medical-surgical setting. However the rubric has potential relevance for other Chinese educational settings and clinical contexts including long-term care and community settings. 


\section{Recommendations}

Faculty need time to become familiar with the use of HPS, spend more time in designing the scenarios and organizing the learning objectives into step-by-step actions. More empirical evidence is needed to validate the importance of the debriefing session in enhancing clinical judgment. Further research is needed to explore whether senior students have better performance in clinical judgment than junior students. The performance indicators associated with the rubric require more research to address content and construct validity in different cultural contexts in order to more accurately reflect the current understanding of each aspect of Tanner's clinical judgment model.

\section{Conclusions}

This study examined one aspect of a clinical education activity in Chinese nursing education. The LCJR offered one way to evaluate students' clinical judgment in a simulated setting. High-fidelity simulation using a computer-controlled human patient simulator offered a realistic learning environment for students to develop their thinking and reasoning skills and has potential to support the development of clinical judgment in nursing students. Tutors are challenged to enhance students' intrinsic motivation for learning, and encourage them to continue their efforts in learning.

\section{References}

[1] Tanner CA. Thinking like a nurse: A research-based model of clinical judgment in nursing. Journal of Nursing Education. 2006; 45(6): 204-211.

[2] Lasater K, Nielsen A. Reflective journaling for clinical judgment development and evaluation. Journal of Nursing Education. 2009; 48(1): 40-44. http://dx.doi.org/10.3928/01484834-20090101-06

[3] Kienle GS, Kiene H. Clinical judgment and the medical profession. Journal of Evaluation in Clinical Practice. 2011; 17(4): 621-627. http://dx.doi.org/10.1111/j.1365-2753.2010.01560.x

[4] Haidar E. Clinical simulation: A better way of learning? Nursing Management. 2009; 16(5): 22-23.

[5] Bambini D, Washburn J, Perkins R. Outcomes of clinical simulation for novice nursing students: Communication, confidence, clinical judgment. Nursing Education Perspectives. 2009; 30(2): 79-82.

[6] Dreifuerst KT. The essentials of debriefing in simulation learning: A concept analysis. Nursing Education Perspectives. 2009; 30(2): 109-114.

[7] Yuan HB, Williams B, Fang JB. The contribution of high-fidelity simulation to nursing students' confidence and competence: a systematic review. International Nursing Review. 2012; 59(1): 26-33. http://dx.doi.org/10.1111/j.1466-7657.2011.00964.x

[8] Yuan HB, Williams B, Fang JB, Ye QH. A systematic review of selected evidence on improving knowledge and skills through high-fidelity simulation. Nurse Education Today. 2012; 32(3): 294-298. http://dx.doi.org/10.1016/j.nedt.2011.07.010

[9] Liao QZ. The application and evaluation of Emergency Care Simulator in pre-job training. Health Occupational Education (Chinese). 2011; 29(3): 84-85.

[10] Ma L, Cai XH, Hu XP. Application of Microsim medical simulation system in experimental teaching of Medical Nursing. China Medical Education Technology. 2010; 24 (1): 69-72.

[11] Lasater K. Clinical judgment development: using simulation to create an assessment rubric. Journal of Nursing Education. 2007; 46(11): 496-503.

[12] Facione NC, Facione PA. Critical Thinking and Clinical Judgment from Critical Thinking and Clinical Reasoning in the Health Sciences: A Teaching Anthology. Millbrae CA: The California Academic Press; 2008.

[13] Rhodes M, Curran C. Use of the human patient simulator to teach clinical judgment skills in a baccalaureate nursing program. CIN: Computers, Informatics, Nursing. 2005; 23(5): 256-262.

[14] Nielsen A, Stragnell S, Jaster P. Guide for reflection using the clinical judgment model. Journal of Nursing Education. 2007; 46(11): 513-516. 